\title{
A simulation study on the relation between muscle motor unit numbers and the non-Gaussianity/non-linearity levels of surface electromyography
}

\author{
ZHAO Yan ${ }^{1,2 *} \&$ LI DongXu ${ }^{1,2}$ \\ ${ }^{1}$ College of Aerospace Science and Engineering, National University of Defense Technology, Changsha 410073, China; \\ ${ }^{2}$ Science and Technology on Human Factors Engineering Laboratory, China Astronaut Research and Training Center, Beijing 100094, China
}

Received August 10, 2012; accepted October 16, 2012

\begin{abstract}
Recent research has demonstrated that surface electromyography (sEMG) signals have non-Gaussianity and non-linearity properties. It is known that more muscle motor units are recruited and firing rates (FRs) increase as exertion increases. A hypothesis was proposed that the Gaussianity test $\left(S_{\mathrm{g}}\right)$ and linearity test $\left(S_{\ell}\right)$ levels of sEMG signals are associated with the number of active motor units (nMUs) and the FR. The hypothesis has only been preliminarily discussed in experimental studies. We used a simulation sEMG model involving spatial (active MUs) and temporal (three FRs) information to test the hypothesis. Higher-order statistics (HOS) from the bi-frequency domain were used to perform $S_{\mathrm{g}}$ and $S_{\ell}$. Multivariate covariance analysis and a correlation test were employed to determine the nMUs- $S_{\mathrm{g}}$ relationship and the nMUs- $S_{\ell}$ relationship. Results showed that nMUs, the FR, and the interaction of nMUs and the FR all influenced the $S_{\mathrm{g}}$ and $S_{\ell}$ values. The nMUs negatively correlated to both the $S_{\mathrm{g}}$ and $S_{\ell}$ values. That is, at the three FRs, sEMG signals tended to a more Gaussian and linear distribution as exertion and nMUs increased. The study limited experiment factors to the sEMG non-Gaussianity and non-linearity levels. The study quantitatively described nMUs and the FR of muscle that are not directly available from experiments. Our finding has guiding significance for muscle capability assessment and prosthetic control.
\end{abstract}

simulation surface electromyography model, higher-order statistics, motor unit, firing rate

Citation: Zhao Y, Li D X. A simulation study on the relation between muscle motor unit numbers and the non-Gaussianity/non-linearity levels of surface electromyography. Sci China Life Sci, 2012, 55: 958-967, doi: 10.1007/s11427-012-4400-1

A motor unit (MU) consists of an alpha motor neuron and connected muscle fibers [1]. An MU structure is made up of a motor neuron, an axon, muscle fibers and a motor endplate [2]. When an MU fires, an action potential is carried down the motor neuron to the muscle. The location where the nerve contacts the muscle is called the motor endplate. After an action potential is transmitted across the motor endplate, an action potential is elicited in all of the innervational muscle fibers of that particular MU. The potential distribution generated in the volume conductor surrounding the fibers of each MU is referred to as "motor unit action

*Corresponding author (email: yanzhao@nudt.edu.cn) potential" (MUAP) [3]. The surface electromyography (sEMG) signal is an algebraic summation of MUAPs [4].

In past research, sEMG signals were usually assumed to be Gaussian with zero mean. However, recent research has indicated that sEMG signals have non-Gaussian and non-linear properties [2,5,6]. Higher-order statistics (HOS) from the bi-frequency domain are able to recover more information from non-Gaussian and non-linear signals via their higher-order moments, cumulants, and spectral representations, and they do not suppress the phase relationship [7]. The HOS approach was recently used to analyze non-Gaussianity and non-linearity sEMG [8]. In specific examples, Bilodeau et al. [5] examined biceps brachii (BB) 
and brachioradialis step and ramp contractions. For the step contractions, four force levels $(20 \%, 40 \%, 60 \%$ and $80 \%$ maximum voluntary contraction (MVC)) were maintained for a period of $3 \mathrm{~s}$ each. Ramp contractions were performed from $0 \%$ to $100 \%$ of MVC in a 5-s period. It was found that the amplitude distributions of both BB and brachioradialis sEMG signals were non-Gaussian, and higher forces gave rise to a slightly more normal sEMG distribution. Applying step contraction tests for a period of $5 \mathrm{~s}$, Nazrpour et al. [9] demonstrated that the non-Gaussianity of BB sEMG signals below $25 \%$ of MVC was significant. Kaplanis et al. [8] found in step contraction tests conducted for a 5-s period that BB sEMG signals tended towards a more Gaussian distribution and a less linear distribution at $70 \%$ of MVC compared with $10 \%, 30 \%, 50 \%$ and $100 \%$ of MVC. Naik et al. [10] conducted step isometric MVC tests at 20\%, 50\%, and $80 \%$ of MVC for a 7-8-s period for flexor digitorum superficialis (FDS). They pointed out that the distribution of FDS sEMG signals tended to be more Gaussian as the force increased, but it also significantly tended towards a Gaussian process below 30\% of MVC. Furthermore, Husssain et al. [11] showed that the distribution of right rectus femoris (RRF) sEMG signals was more Gaussian during slow to fast-walking trials. However, their linearity test results followed the reverse pattern of the Gaussianity test where the RRF signal became more linear in mid-paced walking trials and less linear in a fast-walking trial.

The majority of prior Gaussianity and linearity tests showed that sEMG signals exhibit a more Gaussian distribution as exertion increases. According to the physiological property of the sEMG signal, the central nervous system controls the generation of muscle force by regulating the number of active MUs (nMUs) and the firing rates (FRs) of MUs. As muscle forces increase, more and more MUs and FRs are activated [12]. Several experimental studies have preliminarily discussed that the distribution of sEMG signals is perhaps affected by nMUs $[9,10,13]$. In the present work, a hypothesis is proposed that non-Gaussianity and non-linearity levels for sEMG signals are associated with nMUs and FRs. Reviewing prior Gaussianity and linearity tests of sEMG signals, we found only experimental studies. It is worth noting that different experiments could yield distinct results. Nazrpour et al. [14] suggested that BB sEMG signals became a more "non-Gaussian distribution" below $25 \%$ of MVC, whereas Naik et al. [15] demonstrated that the distribution of FDS sEMG signals was a more "Gaussian distribution" below 30\% of MVC. Possible factors that may lead to these discrepancies include electrode, cable and connector movement, muscle size, exertion level, and cross-talk (a signal contribution originating from other muscles). To limit experimental factors and investigate the change mechanism of the non-Gaussianity and non-linearity levels of sEMG signals in theory, we used a sEMG mathematical model to explore the present assumption. We found no simulated estimation for the non-Gaussianity and non-linearity levels of sEMG signals in the literature.

The purpose of this paper was to test the relationship between nMUs and non-Gaussianity levels for sEMG signals, and the relationship between nMUs and non-linearity levels of sEMG signals at three FRs. An sEMG signal model based on muscle system physiological characteristics was established to simulate nMUs at the three FRs. The HOS approach was used to test the non-Gaussianity and nonlinearity levels of the simulated sEMG signals that were obtained from the sEMG simulation model. This study measured BB muscle activities; BB step contractions were performed at 10 levels from $10 \%$ to $100 \%$ of MVC in a 5-s period. The study initially provides a theoretical supports to quantitatively detect non-linearity and deviations from Gaussianity of the sEMG signal with the MU recruitment number and firing patterns underlying changes. Furthermore, the finding of this study has practical guiding significance for the assessment of muscle activity in the field of occupational medicine and has good potential application for improving prosthetic control in the field of rehabilitation engineering.

\section{Simulation sEMG signal of biceps brachii}

\subsection{Physiological structure of BB}

$\mathrm{BB}$ is the one of the most commonly used muscles. The sEMG of BB can reflect the muscle load and moving angle of the main joint of the individual, i.e., the shoulder and elbow. The study of $\mathrm{BB}$ has representative meaning. As mentioned before, most experiments have focused on $\mathrm{BB}$ muscle in discussing the non-Gaussianity and non-linearity levels of sEMG. We chose BB as the simulated model so that we could fully compare the theory and experiment research. The BB muscle is commonly described as a cylindrical volume conductor. It has a multiple-layer structure and an anisotropic media characteristic [16]. To develop a simulated model of the BB sEMG signal, the BB muscle structure and its parameters must be known (Figure 1). The artificial sEMG signals from BB muscle were hypothetically measured using a bipolar electrode. The interelectrode distance was $20 \mathrm{~mm}$. BB structure input parameters are given in Table 1.

\subsection{Intracellular action potential simulation}

There are two sEMG models. The first model is based on the energy modulation of Gaussian noise, and can be used to analyze the global information of sEMG signals using the amplitude and power spectrum (PS). The second model is based on a physiological property, and can precisely describe MU anatomy [20]. In this paper, we used the second model. Intracellular action potential (IAP) was adopted as the starting point of this sEMG model. IAP is mathemati- 


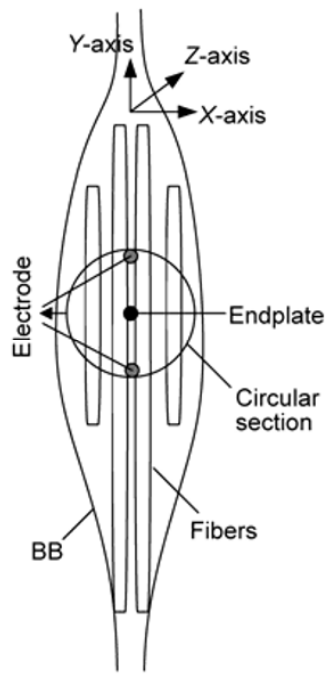

Figure 1 BB structure for the simulation sEMG model.

Table 1 BB structure parameters

\begin{tabular}{|c|c|}
\hline Parameter & Values \\
\hline Muscle area & Circular section \\
\hline $\begin{array}{l}\text { Thickness of the skin and fat } \\
\text { Layer (hs+hf) [16] }\end{array}$ & $4 \mathrm{~mm}$ \\
\hline Fiber diameter $(d)[17,18]$ & $\begin{array}{l}56 \mu \mathrm{m}, \mathrm{SD}=10 \\
\text { Range }= \pm 3 \mathrm{~mm}\end{array}$ \\
\hline Number of fibers in MU ( $n f)$ & 800 \\
\hline Muscle radius [1] & $45-55 \mathrm{~mm}$ \\
\hline Muscle fibers density $(\rho)$ & 20 fibers $\mathrm{mm}^{-2}$ \\
\hline MU size $\left(D_{\mathrm{MU}}\right)[19]$ & $12 \mathrm{~mm}$ \\
\hline $\begin{array}{l}\text { Muscle fiber lengths } L_{0.5 \mathrm{f}} \\
\text { (right+left) [17] }\end{array}$ & $\begin{array}{l}70 \mathrm{~mm}, \text { mean }=0, \\
\mathrm{SD}=1, \text { Range }= \pm 2 \mathrm{~mm}\end{array}$ \\
\hline Fiber-endplate position [18] & Mean $=0$, Range $= \pm 3 \mathrm{~mm}$ \\
\hline Fiber-tendon distance [17] & Range $= \pm 6 \mathrm{~mm}$ \\
\hline Fiber position [16] & Range \pm 1 MU radius \\
\hline MU position [16] & Range \pm 1 muscle radius \\
\hline
\end{tabular}

cally formulated as [21]

$$
e_{i}(z)=768 z^{3} e^{-z}-90,
$$

where $e_{i}(z)$ is fiber IAP; $z$ is the axial direction (Figure 1) in millimeters; and $i$ is an index identifying the MU.

\subsection{Extracellular action potential simulation}

The extracellular action potential (EAP) at an observation point $\left[z_{0}, y_{0}\right]$ can be expressed as [22]

$$
\begin{aligned}
V_{\mathrm{E}}\left(z_{0}, y_{0}\right)= & K\left[\int_{S_{1}} \frac{\partial e(z)}{\partial z} \cdot \frac{1}{r} \mathrm{~d} S+\int_{S} \mathrm{~d} S \int_{-\infty}^{+\infty} \frac{\partial^{2} e(z)}{\partial z^{2}}\right. \\
& \cdot \frac{1}{r} \mathrm{~d} z-\int_{S_{2}} \frac{\partial e(z)}{\partial(z)} \cdot \frac{1}{r} \mathrm{~d} S,
\end{aligned}
$$

where $y$ is the radial direction (Figure 1) in millimeters; $S_{1}$ and $S_{2}$ are the fiber sections at the fiber ends; and $r$ is the distance between the surface element $\mathrm{d} S$ and the observation point:

$$
r=\sqrt{\left(z_{0}-z\right)^{2}+\sigma_{z} / \sigma_{y} y_{0}^{2}} .
$$

Here anisotropy is introduced through $\sigma_{z}$ and $\sigma_{y}$, which denote the axial and radial conductivity, respectively.

The part $I_{m}(z)$ corresponds to the transmembrane current of $K^{\prime \prime}$ :

$$
I_{m}(z)=K^{\prime \prime} \cdot \frac{\partial^{2} e(z)}{\partial z^{2}}=\frac{\pi d^{2} \sigma_{i}}{4} \cdot \frac{\partial^{2} e(z)}{\partial z^{2}}
$$

where $\sigma_{i}$ is the intracellular conductivity.

\subsection{Muscle fiber conduction velocity simulation}

Nandedkar and Stålberg suggested that muscle fiber conduction velocity (MFCV) is related to fiber diameter [21]:

$$
v=2.2+0.05(d-25),
$$

where $v$ is the conduction velocity in meters per second.

\subsection{MU recruitment simulation}

An MU is recruited when input excitation reaches its recruitment threshold. The assigned recruitment threshold excitation $(R T E)$ in the MU pool follows an exponential rule in the form [19]

$$
R T E(i)=\mathrm{e}^{\alpha \cdot i}=\mathrm{e}^{\frac{\ln (R R)}{n} \cdot i},
$$

where $\alpha$ is a coefficient used to establish the range of threshold values; and $R R$ is the range of recruitment threshold values desired. This was set to be a broad MU recruitment range in this paper. That is, the last recruited MU recruitment threshold was set at $70 \%$ of the maximum excitation [23].

\subsection{MU firing simulation}

An MU's peaking firing rate $(P F R)$ is inversely proportional to the recruitment threshold according to [19]

$$
\operatorname{PFR}(i)=\operatorname{PFR}(1)-\operatorname{PFRD} \cdot \frac{\operatorname{RTE}(i)}{R T E(120)}, i=2: 120,
$$

where PFR(1) is the assigned PFR of the first recruited unit; $P F R D$ is the desired difference of the PFR between the first and the last units recruited; $R T E(i)$ is the recruitment threshold of the studied MU; and RTE(120) is the recruitment threshold of the last recruited MU. The MU discharges at a minimum FR of $8 \mathrm{~Hz}$ when the excitatory drive reaches its recruitment threshold. Three FRs were simulated [23]. For FR1, the slope of the excitatory drive-FR relation for an MU increased with increasing recruitment threshold. For FR2, the slope of the excitatory drive-FR relation was set 
the same for all MUs, whereas PFR was linked to the mechanical properties of MUs. For FR3, the slope of the excitatory drive-FR relation was set the same for all MUs. PFR of a MU was inversely proportional to its recruitment threshold.

\subsection{Muscle force generation model}

Muscle force was modeled as an exponential form, linked to the recruitment threshold and varying over a wide range [19]:

$$
F(i)=\mathrm{e}^{\mu \cdot i}=\mathrm{e}^{\frac{\ln (R F)}{n} i},
$$

where $F(i)$ is the force; $\mu$ is a coefficient used to establish a range of twitch force values; and $R F$ is the desired range of twitch force values, which is set to 100 . The step force was simulated as $10 \%, 20 \%, 30 \%, 40 \%, 50 \%, 60 \%, 70 \%, 80 \%$, $90 \%$, and $100 \%$ of MVC.

An sEMG signal simulator was established according to muscle physiological features and the electrode configuration. It is equivalent to the accumulation of 120 MUAPs. Table 2 lists the pertinent parameters from sections 1.2 to 1.7 .

Table 2 MUAP parameters of the simulated sEMG signal

\begin{tabular}{ll}
\hline \multicolumn{1}{c}{ Parameter } & \multicolumn{1}{c}{ Values } \\
\hline Sample rate & $1024 \mathrm{~Hz}$ \\
Muscle contraction period & $5 \mathrm{~s}$ \\
Maximum nMUs [17] & 120 \\
Firing rates [23] & FR1, FR2, FR3 \\
Recruitment threshold [12] & $70 \%$ of maximum excitation \\
MFCV & $3.19 \mathrm{~m} \mathrm{~s}^{-1}$ \\
Minimum FR [12] & $8 \mathrm{~Hz}$ \\
Axial conductivity $\left(\sigma_{z}\right)[17]$ & $0.5 \mathrm{~s} \mathrm{~m}^{-1}$ \\
Radial conductivity $\left(\sigma_{y}\right)[17]$ & $0.1 \mathrm{~s} \mathrm{~m}^{-1}$ \\
Intracellular conductivity $\left(\sigma_{i}\right)[24]$ & $1.01 \mathrm{~s} \mathrm{~m}^{-1}$ \\
\hline
\end{tabular}

\section{Gaussianity test and linearity test}

To perform a bispectrum analysis of HOS, the artificial sEMG signals $x(n)$ were first divided into a series of epochs adjusted to a zero mean value to exclude any signal offset arising from electrode half-cell potentials [25]. The bispectrum analysis is defined as a Fourier transform of the third-order cumulant sequence:

$$
B\left(f_{1}, f_{2}\right)=E\left[X\left(f_{1}\right) X\left(f_{2}\right) X^{*}\left(f_{3}=f_{1} \pm f_{2}\right)\right],
$$

where $X(f)$ is the Fourier transform of time traces of $x(n)$; $E[\cdot]$ is the ensemble expectation; and $f_{i}(i=1,2,3)$ is the wave frequency. There is phase coupling because of non-linear interactions between harmonic components when $k_{1} \pm k_{2}=k_{3}$ and $f_{1} \pm f_{2}=f_{3}$, with ki being the wave number.
To quantify the non-Gaussianity level of a random process, the normalized bispectrum is defined as

$$
B_{\text {norm }}\left(f_{1}, f_{2}\right)=\frac{E\left[X\left(f_{1}\right) \mathrm{X}\left(f_{2}\right) \mathrm{X}^{*}\left(f_{1} \pm f_{2}\right)\right]}{\sqrt{P\left(f_{1}\right) P\left(f_{2}\right) P\left(f_{1} \pm f_{2}\right)}},
$$

where $P(f)$ is the PS.

Consequently, the Gaussianity test is given by

$$
S_{\mathrm{g}}=\sum\left|B_{\text {norm }}\left(f_{1}, f_{2}\right)\right|^{2},
$$

where $S_{\mathrm{g}}$ involves deciding whether the estimated bicoherence is zero. A non-Gaussianity assumption was accepted when the probability of a false alarm (Pfa) is less than $5 \%$ [8]. Linearity test $S_{\iota}$ involves deciding whether the estimated bicoherence is constant in the bi-frequency domain, employing the absolute difference $(\mathrm{d} R)$ between a theoretical $\left(R^{\prime}\right)$ and an estimated inter-quartile range $(R)$. A non-linearity hypothesis was adopted when $\mathrm{d} R / R^{\prime}>2$ [8].

\section{Time-domain and frequency-domain analysis}

The time and frequency domains both fail to consider the non-Gaussianity and non-linearity levels for sEMG signals, and ignore sEMG phase information. However, these two conventional techniques are highly reliable estimators of exertion variation. The root mean square (RMS) from the time domain and the median frequency (MDF) from the frequency domain were also used to characterize BB muscle activities [26]. RMS is defined as

$$
R M S=\sqrt{\frac{1}{N} \sum_{j=1}^{N} x_{j}^{2}},
$$

where $x_{j}$ is the $j$ th sample of a signal; and $N$ is the number of samples in the epoch.

MDF is computed using a 512-point Hanning window:

$$
\int_{0}^{f_{\text {med }}} P(f) \mathrm{d} f=\frac{1}{2} \int_{0}^{f_{\mathrm{s}} / 2} P(f) \mathrm{d} f,
$$

where $f_{\text {med }}$ is the MDF; and $f_{\mathrm{s}}$ is the sampling frequency.

\section{Results}

\section{1 sEMG simulation results}

Figure 2 illustrates the artificial BB sEMG signals from $10 \%$ to $100 \%$ of MVC for the three FRs. To conveniently compare the amplitudes of sEMG signals, the signals are shown in normalized form. The blue long-dash lines, redsolid lines, and green short-dash lines present sEMG signals for FR1, FR2 and FR3, respectively. The sEMG signal amplitudes of the three FRs evidently broadened from $10 \%$ to $100 \%$ of MVC. 


\subsection{Gaussianity test and linearity test results}

Figure 3 shows the Pfa values and $S_{\mathrm{g}}$ values of the Gaussianity test, and the $S$ c values of the linearity test for the three FRs. The horizontal ordinate in Figure 3 reflects that nMUs increased as the MVC level increased. For the three FRs, the sEMG signals for nMUs were $65,84,96,104,110,115$, $120,120,120$ and 120 corresponding to the 10 levels of MVC. Viewing the curves in Figure 3, most Pfa values were less than 5\%. Several Pfa values were more than $5 \%$ and
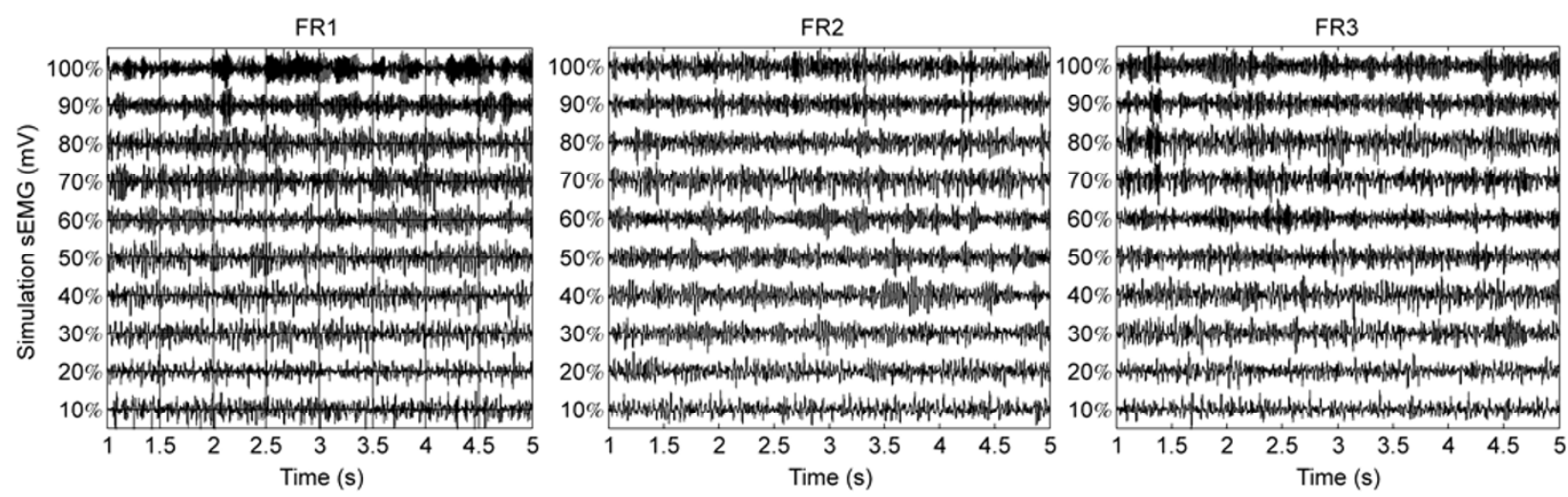

Figure 2 sEMG signals from $10 \%$ to $100 \%$ of MVC for the three FRs.
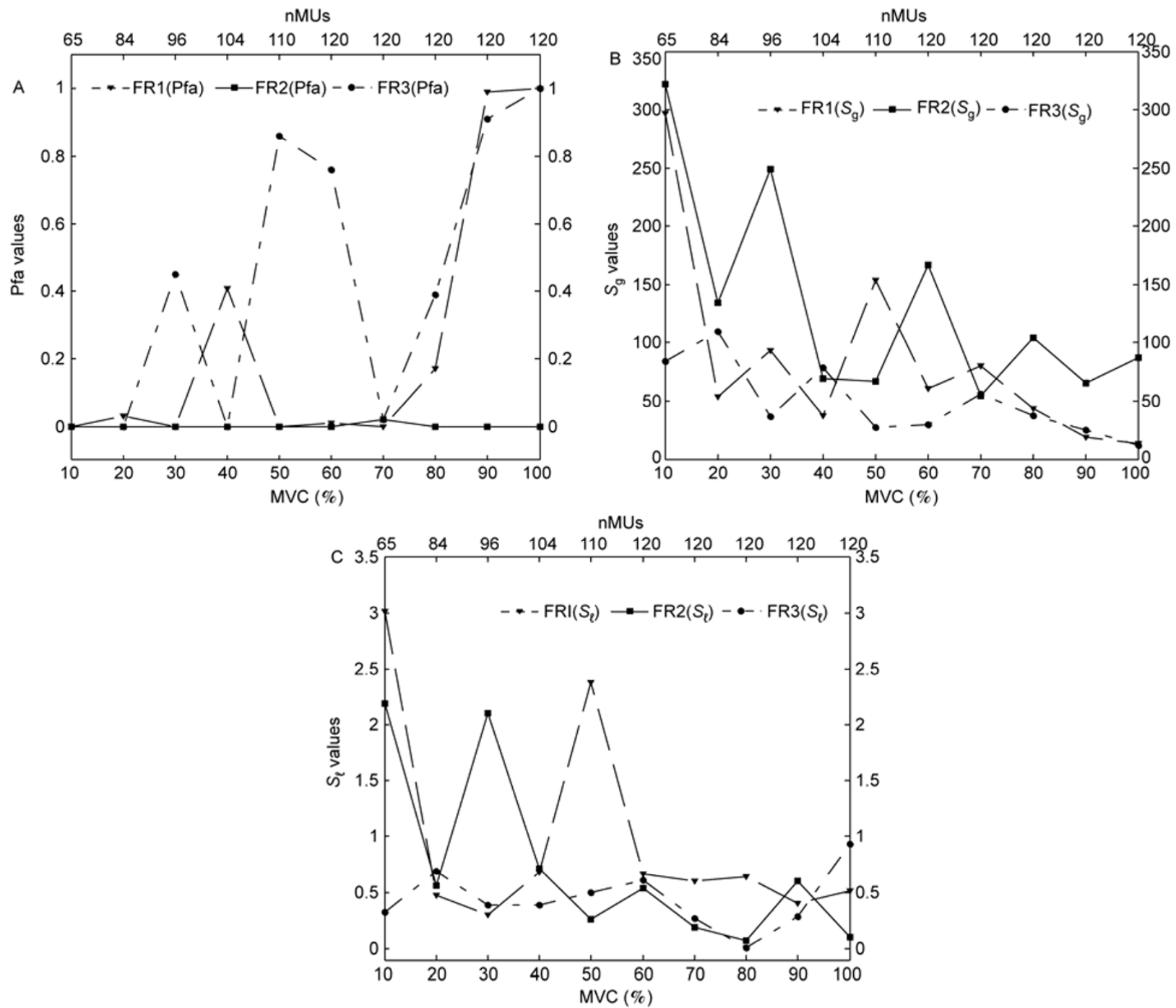

Figure 3 Gaussianity and linearity tests for the three FRs. A, Pfa values of the Gaussianity test. B, $S_{\mathrm{g}}$ values of the Gaussianity test. C, $S_{\ell}$ values of the linearity test. 
were approximately 1 (Table 3 ). Most $\mathrm{d} R / R^{\prime}$ values were smaller than 2 . The $\mathrm{d} R / R^{\prime}$ values that are much larger than 2 are listed in Table 4. As MVC levels and nMUs increased, the $S_{\mathrm{g}}$ and $S_{\ell}$ results of sEMG signals for the three FRs all declined sharply.

\subsection{Multivariate analysis of covariance test results}

A multivariate analysis of covariance (MANOVA) was conducted to test the effects of nMUs and FRs on the non-Gaussianity and non-linearity levels of the sEMG signal. In Table 5, $\mathrm{d} f$ is the number of degrees of freedom; $F$ is the $F$-statistics; and $P$ is the $P$-value probability. The dependent variables are $S_{\mathrm{g}}$ and $S_{\ell}$, and the independent variables are nMUs and the FR. nMUs and the FR significantly affected $S_{\mathrm{g}}$ and $S_{\ell}$ at the $P=0.000$ level. Interaction between nMUs and the FR also affected the $S_{\mathrm{g}}$ and $S_{\ell}$ at the $P=0.003$ and $P=0.000$ levels, respectively. MANOVA gives four results: (i) there were differences in the Gaussianity and linearity test results for the sEMG signal because of the change in the FR when nMUs was held constant; (ii) there were differences in the sEMG signal Gaussianity and linearity test results because of the change in nMUs when the FR was held constant; (iii) the interactive relationship be-

Table 3 Gaussianity test Pfa values that are approximately 1

\begin{tabular}{ccc}
\hline Strategies & MVC & Pfa values \\
\hline \multirow{2}{*}{ FR1 } & $90 \%$ & 0.99 \\
& $100 \%$ & 1.00 \\
\multirow{2}{*}{ FR3 } & $50 \%$ & 0.86 \\
& $60 \%$ & 0.76 \\
& $90 \%$ & 0.91 \\
& $100 \%$ & 1.00 \\
\hline
\end{tabular}

Table 4 Linearity test $\mathrm{d} R / R^{\prime}$ values that are more than 2

\begin{tabular}{ccc}
\hline Strategies & MVC & $\mathrm{d} R / R^{\prime}$ values \\
\hline \multirow{2}{*}{ FR1 } & $10 \%$ & 3.01 \\
& $50 \%$ & 2.38 \\
\multirow{2}{*}{ FR2 } & $10 \%$ & 2.19 \\
& $30 \%$ & 2.1 \\
\hline
\end{tabular}

Table 5 MANOVA test results for the three FRs

\begin{tabular}{cccrc}
\hline Source & Dependent variables & $\mathrm{d} f$ & \multicolumn{1}{c}{$F$} & $P$ \\
\hline Corrected & $S_{\mathrm{g}}^{\mathrm{a}}$ & 20 & 14.90 & 0.000 \\
model & $S_{\ell}{ }^{\mathrm{b})}$ & 20 & 60.40 & 0.000 \\
& $S_{\mathrm{g}}$ & 6 & 25.14 & 0.000 \\
nMUs & $S_{\ell}$ & 6 & 104.10 & 0.000 \\
& $S_{\mathrm{g}}$ & 2 & 32.32 & 0.000 \\
FRs & $S_{\ell}$ & 2 & 72.29 & 0.000 \\
nMUs & $S_{\mathrm{g}}$ & 12 & 7.41 & 0.003 \\
$*$ FRs & $S_{\ell}$ & 12 & 40.47 & 0.000 \\
\hline
\end{tabular}

a) $R^{2}=0.971$ (adjusted $\left.R^{2}=0.906\right)$. b) $R^{2}=0.993$ (adjusted $\left.R^{2}=0.976\right)$. tween nMUs and the FR affected the Gaussianity and linearity test results for the sEMG signal; and (iv) the FR, nMUs, the interactive effects between the FR and nMUs, and test errors were the significant predictor, could explain $97.1 \%$ and $99.3 \%$ of the total variance of Gaussianity test results and linearity test results, respectively (see footnotes $\mathrm{a}$ and $\mathrm{b}$ in Table 5).

\subsection{Bivariate correlation test results}

A bivariate correlation test was carried out to determine the correlation between nMUs and $S_{\mathrm{g}}$, and the correlation between nMUs and $S_{\ell}$ for the three FRs. The Pearson correlation coefficients in Table 6 indicate that the nMUs- $S_{\mathrm{g}}$ relationship and the nMUs- $S_{\ell}$ relationship both have negative correlation. In addition, the $S_{\mathrm{g}}-S_{\ell}$ relationship has positive correlation. The highest correlation $(r=-0.83)$ was between nMUs and $S_{\ell}$ for FR2 $(P<0.01)$. All Pearson coefficients for FR1 and FR2 were clearly significant $(P<0.05)$. The correlation coefficients for the nMUs- $S_{\ell}$ relation and those for the $S_{\mathrm{g}}-S_{\ell}$ relation for FR3 were very low (see footnotes $*$ and $* *$ in Table 6).

\subsection{Time-domain and frequency-domain results}

RMS and MDF results of the simulated sEMG signals for the three FRs are summarized in Figure 4. RMS increased markedly as the force and nMUs increased. MDF results did not shifted to the lower frequencies with muscle force and nMUs gradually increasing. In contrast, MDF results for the three FRs slightly shifted to the higher frequencies for all levels of exertion.

\section{Discussion}

The purpose of this study was to test the hypothesis that the non-Gaussianity and non-linearity levels of sEMG signals are associated with nMUs at three FRs. Prior experimental studies concluded that the non-Gaussianity and non-linearity levels of sEMG signals change with muscle force levels. However, the change mechanism of sEMG non-Gaussianity and non-linearity levels for the nMUs and FR strategies of

Table 6 Bivariate correlation test results for the three FRs ${ }^{\text {a) }}$

\begin{tabular}{ccccc}
\hline Parameters & nMUs & FR1 $\left(S_{\mathrm{g}}\right)$ & FR2 $\left(S_{\mathrm{g}}\right)$ & FR3 $\left(S_{\mathrm{g}}\right)$ \\
\hline nMUs & 1 & & & \\
FR1 $\left(S_{\mathrm{g}}\right)$ & $-0.74^{*}$ & 1 & & \\
FR2 $\left(S_{\mathrm{g}}\right)$ & $-0.79^{* *}$ & & 1 & 1 \\
FR3 $\left(S_{\mathrm{g}}\right)$ & $-0.73^{*}$ & & & \\
FR1 $\left(S_{\ell}\right)$ & $-0.69^{*}$ & $0.96^{* *}$ & & \\
FR2 $\left(S_{\ell}\right)$ & $-0.83^{* *}$ & & $0.93^{* *}$ & \\
FR3 $\left(S_{\ell}\right)$ & -0.40 & & & 0.49 \\
\hline
\end{tabular}

a) *, $P<0.05 ; * *, P<0.01$. 

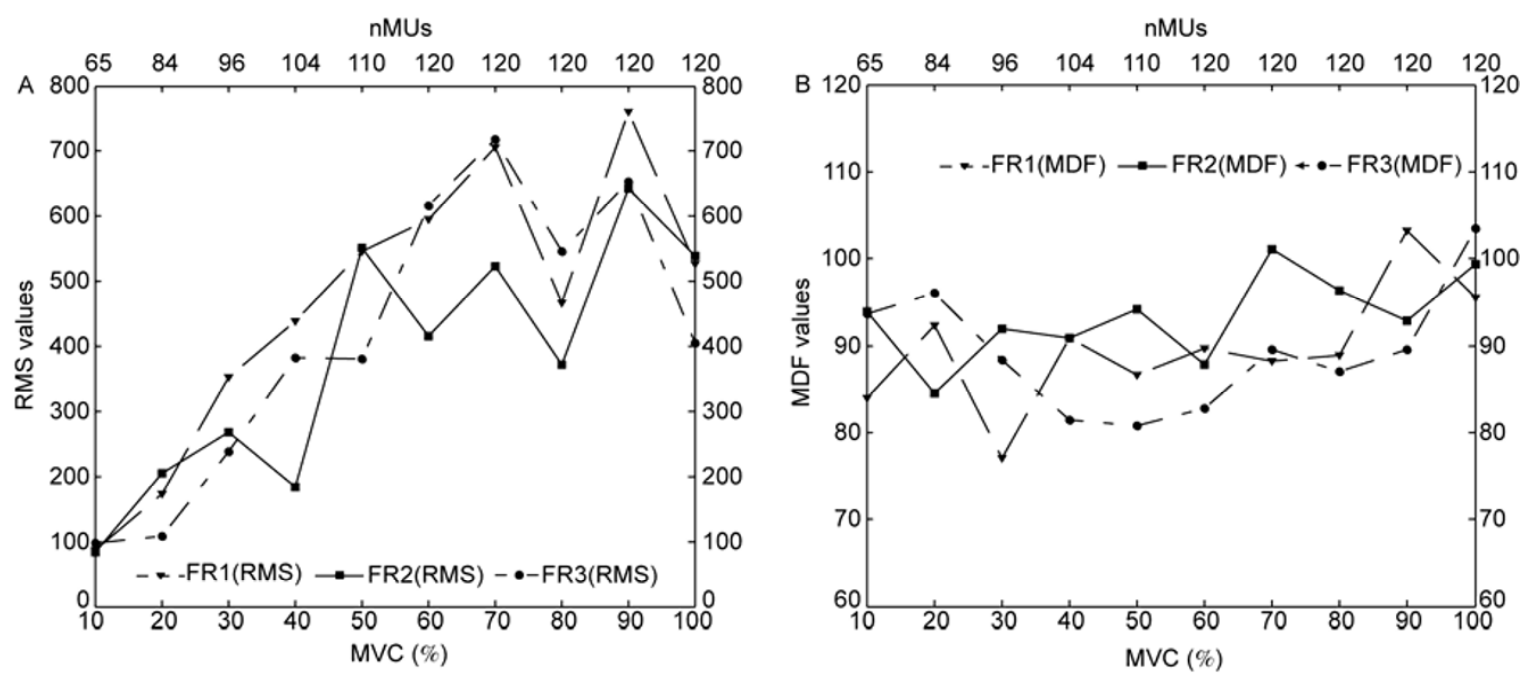

Figure 4 Time-domain and frequency-domain results for the three FRs. A, RMS values of time-domain analysis. B, MDF values of frequency-domain analysis.

muscle is not directly available from experimental studies, and remains elusive. Thus, we used a mathematical simulated model to investigate the present hypothesis in theory. This study quantitatively shed light on the relation between nMUs and the non-Gaussianity levels of sEMG signals, and the relation between nMUs and the non-linearity levels of the sEMG signal for the three FRs. The current effort may stimulate further work on two fronts. First, it can be used to objectively and comprehensively evaluate muscle capability in a normal individual and an individual suffering from a neuromuscular disorder. Second, it can be applied in the control of prosthetics for an individual with an amputation or congenitally deficient limb. The mathematical simulated model can avoid the effects of real physical world factors, such as electrode movement and cross-talk. It was developed according to musculoskeletal system physiology characteristics. The model deduced sEMG signals involving spatial (active MUs) and temporal (FR) information. The levels of the non-Gaussianity and non-linearity of simulated sEMG signals were estimated using the HOS approach. A MANOVA and a bivariate correlation test were used to quantify the test results for the nMUs-Gaussianity relationship, the nMUs-linearity relationship, and the Gaussianitylinearity relationship. The study contributes three major findings.

First, the horizontal ordinate in Figure 3 shows that the nMUs for the three FRs had the same incremental trend as exertion increased. The BB sEMG signals for all three FRs achieved full MU recruitment at 70\% MVC. From eq. (6), we know that nMUs depend on the exertion level and $R R$, and are independent of the FR strategy. The FR does not affect nMUs at each exertion level. All MUs were recruited at $70 \%$ of MVC because the MU recruitment range was set at $70 \%$ maximum excitation.

Second, Figure 3 shows that $S_{\mathrm{g}}$ and $S_{\ell}$ significantly de- crease as forces and nMUs increase. $S_{\mathrm{g}}$ is estimated in a $\chi^{2}$ distribution test. The principle of the $\chi^{2}$ distribution test is

$$
\chi^{2}=\sum \frac{\left(f_{0}-f_{\mathrm{e}}\right)^{2}}{f_{\mathrm{e}}},
$$

where $f_{0}$ is the observed frequency; and $f_{\mathrm{e}}$ is the theoretical frequency. For a smaller value of $S_{\mathrm{g}}$, there is little difference between $f_{0}$ and $f_{\mathrm{e}}$. In this paper, $f_{0}$ is the frequency distribution of the sEMG signals; and $f_{\mathrm{e}}$ is the theoretical Gaussian frequency distribution. It is clear that nMUs increased and $S_{\mathrm{g}}$ decreased as the force increased for all three FRs. Thus, the Gaussianity test revealed that sEMG signals tended toward a more Gaussian distribution as the force and nMUs increased at all three FRs. The non-Gaussianity of sEMG signals below $40 \%$ MVC was significant for all three FRs.

The linearity of sEMG signals was calculated as $\left|R^{\prime}-R\right|$. Here, $R^{\prime}$ corresponds to $\chi_{2}^{2}(\lambda)$, that is, a $\chi^{2}$ distributed random variable having two degrees of freedom and a non-centrality parameter. $R$ is derived from the estimated squared bicoherence. In Figure 3C, nMUs increased and $\mathrm{d} R$ decreased as the force increased at all three FRs. The linearity test of sEMG signals revealed that sEMG signals were a more linear distribution as the force increased at all three FRs. Additionally, the linearity of sEMG signals above $60 \%$ of MVC was significant at all three FRs.

In Table 3, the Pfa values for FR1 at $90 \%$ and $100 \%$ of MVC, and for FR3 at $50 \%, 60 \%, 90 \%$, and $100 \%$ of MVC were approximately 1 . We accepted the Gaussian hypothesis at these MVC levels. In Table 4 , the $\mathrm{d} R / R^{\prime}$ values for FR1 at $10 \%$ and $50 \%$ of MVC, and for FR2 at $10 \%$ and $30 \%$ of MVC were more than 2. We accepted the non-linear hypothesis at these MVC levels. Furthermore, all Pfa values for FR2 were less than $5 \%$, and all $\mathrm{d} R / R^{\prime}$ values for FR3 were less than 2 . In virtue of the indeterminable nature and 
complexity of human muscle activities, and the unknown precise motoneuron FR distribution for different MUs, we speculate that the non-Gaussianity and non-linearity discrepancy among the three FRs was caused by different PFR values for the three FRs (eq. (7)). For FR1, all MUs finally reached the same $P F R$ at $70 \%$ of MVC (Figure 5A). In the FR2 simulation, the $P F R$ values were linked to the mechanical properties of MUs. That is, later-recruited large rapidly contracting units were assigned higher PFRs than were those with lower recruitment thresholds and with small twitches and slow contraction times (Figure 5B). In FR3 simulation, slope of the excitatory drive-FR relation was set to be the same for all MUs. The PFR of an MU was inversely proportional to its recruitment threshold (Figure 5C) [23]. The three-FR waveforms altered the sEMG signal distribution.

Finally, the correlation between nMUs and non-Gaussianity, and the correlation between nMUs and the nonlinearity, were quantitatively analyzed at the three FRs. The MANOVA test and bivariate correlation test were performed to shed light on our hypothesis (see results in Tables 5 and 6). The MANOVA test validated that not only the two main factors nMUs and the FR but also interaction between these factors was responsible for the changes in the non-Gaussianity and non-linearity levels of sEMG signals. The bivariate correlation test demonstrated that nMUs was negatively correlated to both the Gaussianity and linearity results of sEMG signals. Furthermore, for all three FRs, the Gaussianity test results of sEMG signals was positively correlated to the linearity test results.

Similarly, Bilodeau et al. [5] and Kaplanis et al. [8] both reported that BB sEMG signals tended to be a more Gaussian distribution as exertion increased. Nazarpour et al. [9] found that the non-Gaussianity of BB sEMG signals below $25 \%$ of MVC was significant. Naik et al. [10] found that FDS sEMG signals tended towards a Gaussian distribution as exertion increased. However, they also pointed out that FDS sEMG signals tended to be a more Gaussian distribution below $30 \%$ of MVC. The later finding of Naik is the converse of our result. The discrepancy may stem from the difference in MU recruitment between small and large muscles. Small muscles have been reported to have narrow MU recruitment, such as in the case of FDS. Large muscles have a mixed fiber composition. They are generally regarded as having a broad MU recruitment range, such as the case for $\mathrm{BB}$ [23]. Although the FR and MU recruitment both contribute to force production, the FR plays a more important role in small muscles and MU recruitment is important in large muscles [27]. Small muscle MUs obey the so-called "size principle" [28], which states that MUs are activated in order from the smallest to the largest MU. This "size principle" does not explain the behavior of large muscles. Thus, we argue that the MU recruitment range and "size principle" explain the difference between small and large muscles.

The sEMG linearity test carried out by Kaplanis et al. [8] revealed that $\mathrm{BB}$ sEMG signals exhibited more Gaussianity at $70 \%$ of MVC, while sEMG signals showed less linearity at $70 \%$ of MVC. A more recent study by Hussian et al. [11] performed sEMG signal linearity tests for the RRF. They reported that the linearity test followed the reverse pattern of the Gaussianity test. Unlike these linearity results, our linearity test results were positively correlated to the Gaussianity results. We tentatively argue that this apparent conflict results from muscle fatigue. In time and frequency domains, if the EMG amplitude increases and the EMG spectrum shifts to the right (i.e., the time-domain and frequency-domain curves turn upward), then the probable cause is an increase in muscle force. If the EMG amplitude increases and the EMG spectrum shifts to the left (i.e., the time-domain curve turns upward and the frequency-domain curve turns downward), then this is considered to be a result of muscle fatigue [14]. The results in the time and frequency domains for the three FRs are shown in Figure 4. The sEMG signal RMS values increased, and simultaneously the MDF values slightly shifted to the higher frequencies. Thus, our sEMG signal variation is related to the force. Timedomain and frequency-domain analyses were carried out
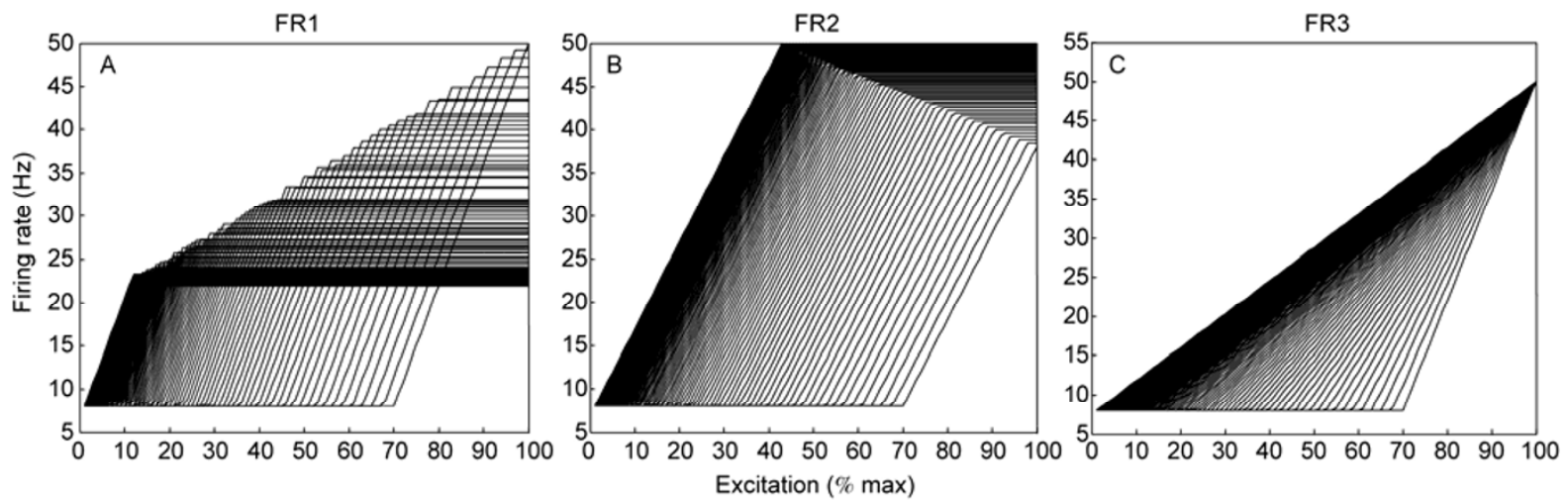

Figure $5 \mathrm{BB}$ broad recruitment simulation for the three FRs. 
by Kaplanis et al. and Hussian et al., who found that the time-domain outputs increased and the frequency-domain outputs shifted to the lower frequencies as force level increased. The sEMG signal variations of those two studies were described as fatigue-induced. We deduce that sEMG signals have a more Gaussian and more linear distribution in the bi-frequency domain probably because of an increase in large muscle force, and they have a more Gaussian and less linear distribution probably because of large muscle fatigue.

\section{Conclusion}

The simulated sEMG signal model in the present research was used to quantitatively test the relation between nMUs and the non-Gaussianity levels of sEMG signals, and the relation between nMUs and non-linearity levels of the sEMG signal for three FRs. Results showed that the BB sEMG signal was a more Gaussian and more linear distribution as nMUs and exertion increased. The non-Gaussianity level of the sEMG signal below $40 \%$ MVC is significant, and the linearity level of the sEMG signal above $60 \%$ MVC is significant. The changes in non-Gaussianity and non-linearity levels of the sEMG signal essentially rely on nMUs and FRs.

This work was supported by the National High Technology Research and Development Program of China and the National Basic Research Program of China (Grant No. 2011CB7000).

\section{Abbreviations}

$\begin{array}{ll}\text { Biceps Brachii } & \text { BB } \\ \text { Extracellular Action Potential } & \text { EAP } \\ \text { Firing Rate } & \text { FR } \\ \text { Flexor Digitorum Superficialis } & \text { FDS } \\ \text { Higher-order Statistics } & \text { HOS } \\ \text { Intracellular Action Potential } & \text { IAP } \\ \text { Motor unit } & \text { MU } \\ \text { Median Frequency } & \text { MDF } \\ \text { Maximum Excitation } & \text { ME } \\ \text { Motor Unit Action Potential } & \text { MUAP } \\ \text { Maximum Voluntary Contraction } & \text { MVC } \\ \text { Muscle Fiber Conduction Velocity } & \text { MFCV } \\ \text { Multivariate Analysis of Covariance } & \text { MANOVA } \\ \text { Number of active MUs } & \text { nMUs } \\ \text { Peaking Firing Rate } & \text { PFR } \\ \text { Power Spectrum } & \text { PS } \\ \text { Probability of False Alarm } & \text { Pfa } \\ \text { Right Rectus Femoris } & \text { RRF } \\ \text { Root Mean Square } & \text { RMS } \\ \text { Recruitment Threshold Excitation } & \text { RTE }\end{array}$

$\begin{array}{ll}\text { Surface electromyography } & \text { sEMG } \\ \text { Gaussianity test } & S_{\mathrm{g}} \\ \text { Linearity test } & S_{\ell}\end{array}$

1 Buchthal F, Erminio F, Rosenfalck P. Motor unit territory in different human muscles. Acta Physiol Scand, 1959, 45: 72-87

2 Merletti R, Rainoldi A, Farina D. Electromyography: Physiology, Engineering and Noninvasive Application. New Jersey: John Wiley \& Sons, 2004

3 Merletti R, Roy S H, Kupa E, et al. Modeling of surface myoelectric signals-Part II: model-based signal interpretation. IEEE Trans Biomed Eng, 1999, 46: 821-829

4 Day S J, Hulliger M. Experimental simulation of cat electromyogram: evidence for algebraic summation of motor-unit action-potential trains. J Neurophysiol, 2001, 86: 2144-2158

5 Bilodeau M, Cincera M, Arsenault A B, et al. Normality and stationarity of EMG signals of elbow flexor muscles during ramp and step isometric contractions. J Electromyogr Kines, 1997, 7: 87-96

6 Zazula D. Experience with surface EMG decomposition using higher-order cumulants. In Proceedings of Signal Processing 2001 of IEEE Workshop, Poznãn, Poland, 2001. 19-24

7 Shahid S, Walker J, Lyons G M, et al. Application of higher order statistics techniques to EMG signals to characterize the motor unit action potential. IEEE Trans Biomed Eng, 2005, 52: 1195-1209

8 Kaplanis P A, Pattichis C S, Hadjileontiadis L J, et al. Surface EMG analysis on normal subjects based on isometric voluntary contraction. J Electromyogr Kines, 2009, 19: 157-171

9 Nazarpour K, Sharafat A R, Firoozabadi S M P. Application of higher order statistics to surface electromyogram signal classification. IEEE Trans Biomed Eng, 2007, 54: 1762-1769

10 Naik G, kumar D. Evaluation of higher order statistics parametes for multi channel sEMG using different force levels. In: 33rd Annual International Conference of the IEEE, Boston, USA, 2011. 3869-3872

11 Hussain M S, Mamun M D. Effectiveness of the wavelet transform on the surface EMG to understand the muscle fatigue during walk. Meas Sci Rev, 2012, 12: 28-33

12 Zhou P, Rymer W Z. Can standard surface EMG processing parameters be used to estimate motor unit global firing rate? J Neural Eng, 2004, 1: 99-110

13 Kernell D. Organized variability in the neuromuscular system: a survey of task-related adaptations. Arch Ital Biol, 1992, 130: 1966

14 Staudenmann D, Roeleveld K, Stegeman D F, et al. Methodological aspects of sEMG recordings for force estimation-A tutorial and review. J Electromyogr Kines, 2009, 20: 375-387

15 Turker K S, Miles T S. Cross-talk from other muscles can contaminate EMG signals in reflex studies of the human leg. Neurosci Lett, 1990, 111: 164-169

16 Wang W, Stefano A D, Allen R. A simulation model of the surface EMG signal for analysis of muscle activity during the gait cycle. Comput Biol Med, 2006, 36: 601-618

17 Gabriel D A, Kamen G. Experimental and modeling investigation of spectral compression of biceps brachii SEMG activity with increasing force levels. J Electromyogr Kines, 2009, 19: 437-448

18 Duchêne J, Hogrel J Y. A model of EMG generation. IEEE Trans Biomed Eng, 2000, 47: 192-201

19 Fuglevand A J, Winter D A, Patla A E. Models of recuitment and rate coding organization in motor-unit pools. J Neurophysiol, 1993, 70: 2470-2488

20 McGill K C. Surface electromyogram signal modelling. Med Biol Eng Comput, 2004, 42: 446-454

21 Nandedkar S D, Stålberg E. Simulation of sigle fiber action potentials. Med Biol Eng Comput, 1983, 21: 158-165

22 LorentedeNó R. Analysis of the distribution of action currents of nerve in volume conductors. Stud Rockfeller Inst Med Res, 1974, 132: $384-477$ 
23 Zhou P, Rymer W Z. Factor governing the form of the relation between muscle force and the EMG: A simulation study. J Neurophysiol, 2004, 92: 2878-2886

24 Andreassen S, Rosenfalck A. Relation of intracellular and extracellular action potentials of skeletal muscle fibers. OCR Crit Rev Biomed Eng, 1981, 6: 267-306

25 Sigl J C, Chamoun N C. An introduction to bispectral analysis for the electroencephalogram. J Clin Monit, 1994, 10: 392-404
26 Zhou Q X, Chen Y H, Ma C, et al. Evaluation of upper limb muscle fatigue based on surface electromyography. Sci China Tech Sci, 2011, 54: 939-944

27 DeLuca C J, LeFever R S, McCue M P, et al. Behaviour of human motor units in different muscles during linearly varying contractions J Physiol, 1982, 329: 113-128

28 Henneman E. Relation between size of neurons and their susceptibility to discharge. Science, 1957, 126: 1345-1347

Open Access This article is distributed under the terms of the Creative Commons Attribution License which permits any use, distribution, and reproduction in any medium, provided the original author(s) and source are credited. 